\title{
Identificación de helmintos en carpa común (Cyprinus carpio) de un ecosistema acuático en el norte de México
}

\author{
Identification of helminthes in common carp (Cyprinus \\ carpio) from an aquatic environment in the north of Mexico
}

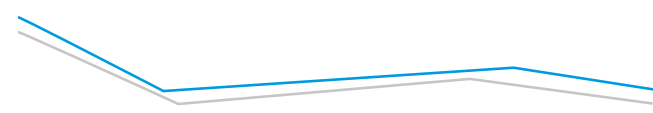

Héctor Rubio Arias ${ }^{1 *}$, Magali Domínguez Chacón', Martín Renato Hernández Castaños², María Eduviges Burrola Barraza', Luis Colmenero³, María de Lourdes Villalba ${ }^{4}$

Rubio Arias, H., Domínguez Chacón, M., Hernández Castaños, M. R., Burrola Barraza, M. E., Colmenero L., Villalba, M. L. Identificación de helmintos en carpa común (Cyprinus carpio) de un ecosistema acuático en el norte de México. Investigación y Ciencia de la Universidad Autónoma de Aguascalientes. Número 68: 15-21, mayo-agosto 2016.

\section{RESUMEN}

El pescado es un alimento imprescindible en la dieta humana, debido a que su consumo provee proteínas, lípidos, sales minerales, carbohidratos y vitaminas. Sin embargo, el parasitismo en los peces es un fenómeno que puede afectar a la salud humana. El objetivo del trabajo fue identificar la morfología de helmintos en carpa común (Cyprinus carpio) procedentes de la Laguna de Bustillos en Chihuahua, México. En otoño de 2011 se colectaron 30 especímenes $(N=30)$ y se realizó un análisis parasitoscópico determinando la frecuencia total parasitaria (FT) y la frecuencia parasitaria por especie (FP). Se encontró una FT de $90 \%$, en la que Gyrodactylus spp. se localizó en agallas, Pseudocapillaria tomentosa en intestino y Botriocephalus acheilognathi parasitando la mucosa intestinal. Se observó una FP de $P$. tomentosa con $83.33 \%$, de Gyrodactylus spp. con $76.67 \%$ y de B. acheilognathi con $6.67 \%$. Se recomienda que el consumidor realice la ingesta de pescado atendiendo medidas sanitarias como su preparación

Palabras clave: carpa común, análisis parasitoscópico, Gyrodactylus, Pseudocapillaria, Botriocephalus.

Keywords: common carp, parasitological analysis, Gyrodactylus, Pseudocapillaria, Botriocephalus.

\footnotetext{
Recibido: 25 de marzo de 2015, aceptado: 21 de marzo de 2016

Facultad de Zootecnia y Ecología, Universidad Autónoma de Chihuahua. Facultad de Ciencias Químicas, Universidad Autónoma de Chihuahua. Instituto Tecnológico de Chihuahua II.

Facultad de Ingeniería, Universidad Autónoma de Chihuahua.

Autor para correspondencia: rubioa1105@hotmail.com
}

a $60^{\circ} \mathrm{C}$ por más de 10 min para matar las larvas que pudieran ser zoonóticas al ser humano.

\section{ABSTRACT}

Fish constitute an essential part of human diet, because its consumption provides important nutrients such as protein, lipids, mineral salts, carbohydrates and vitamins. However, despite these benefits, it has been discovered that parasitism in fish is a common phenomenon that represents a potentially risk for human health. Therefore, the study of the presence of helminthes in individuals of common carp (Cyprinus carpio) present in the Laguna de Bustillos, Chihuahua, Mexico, was established as the objective of this research. During the period from September to November 2011, a total of 30 fishes ( $N=30$ ) were captured and summated to a parasitoscopic analysis that allowed obtaining the total frequency of parasitism (FT) and frequency of parasitism (FP) by specie were determined. The results showed up a FT of $90 \%$, finding Gyrodactylus spp. in gills, Pseudocapillaria tomentosa in the intestine and Botriocephalus acheilognathi in the intestinal mucosa from the fish specimens studied. The FP obtained was about $83.33 \%$ for P. tomentosa, about $76.67 \%$ for Gyrodactylus spp. and $6.67 \%$ for B. acheilognathi. Due to the high frequency of helminthes found in common carp, it is highly recommended that the consumption of these fish to be after the necessary sanitary measures, such as cooking the fish above 60 ${ }^{\circ} \mathrm{C}$ for over $10 \mathrm{~min}$; action that will allow the elimination of larvae which may be zoonotic to humans. 


\section{IIVESEIGACIÓn

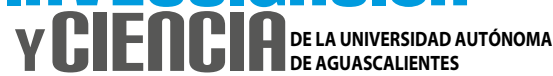

INTRODUCCIÓN

El ecosistema conocido como Laguna de Bustillos es un cuerpo de agua natural localizado en el noroeste del estado de Chihuahua, México. Algunos estudios realizados en este ecosistema endorreico han reportado niveles de contaminación en el agua con diversos elementos como Fe (hierro), Mn (manganeso), $\mathrm{Ni}$ (níquel), V (vanadio), Zn (zinc) y Li (litio) (Rubio et al., 2005) en sedimentos (Soto, 2011) e incluso, en los suelos de sus alrededores con los metales pesados $\mathrm{Cd}$ (cadmio) y Pb (plomo) (Rubio et al., 2006). Esta contaminación se explica por los desechos vertidos de la industria Celulosa Chihuahua, de dos grandes ciudades como Cuauhtémoc y Anáhuac y por la escorrentía de la actividad agrícola-pecuaria de grupos menonitas, que van a ser almacenados en este ecosistema. Como consecuencia, se puede hipotetizar que su ictiofauna ha sido afectada.

Los peces, al igual que la mayoría de los seres vivos, están expuestos a diversas infecciones; sin embargo, las enfermedades parasitarias no se manifiestan excepto cuando las condiciones del hospedero, o bien del medio ambiente, permiten su proliferación. En cualquier caso, el parasitismo es un fenómeno constante en los peces (Kinkelin et al., 1991). Ha sido bien documentado que existen trastornos parasitarios en el ser humano que inician a través del contacto directo con los animales, o bien, mediante ingesta de carne infectada; a esta transmisión de origen animal-ser humano se le identifica como zoonosis (Slifko et al., 2000; Becerril, 2008).

Es importante la identificación de parásitos que afectan la ictiofauna de un determinado cuerpo de agua, en especial cuando los peces son destinados para el consumo humano, ya que pueden causar enfermedades zoonóticas, parasitosis médica y, en el peor de los casos, parasitosis clínica. Es importante mencionar que una de las especies de peces más importantes en México es la carpa común (Cyprinus carpio), una especie introducida (FAO, 2007) y, a la fecha, se le considera también como una de las principales en los ecosistemas acuáticos del norte de México.

El objetivo de este estudio fue identificar la morfología de helmintos en carpa común (C. carpio) que se desarrolla en la Laguna de Bustillos, Chihuahua, México. Para el caso particular de
México han sido documentadas 262 especies de helmintos en 152 géneros y 59 familias catalogadas en tres grupos taxonómicos (Salgado Maldonado, 2006), la mayoría identificados en el sur de México (Salgado Maldonado et al., 2014a), pero a la fecha no se conoce información sobre los parásitos que infectan a la carpa común que se desarrolla en la Laguna de Bustillos. Esta exploración es relevante dado que los habitantes consumen pescado procedente de dicho cuerpo de agua de manera importante en su dieta diaria. Los resultados de este estudio permitirán dar a conocer información a los consumidores rurales, así como a las autoridades de todos los niveles en México para prevenir o corregir daños potenciales al ecosistema y a la salud humana.

\section{MATERIALES Y MÉTODOS}

\section{Obtención de especímenes, sacrificio y disección}

La presente investigación consistió en dos fases. La primera implicó la obtención de especímenes vivos, los cuales fueron capturados en la Laguna de Bustillos (28 33' 16.00" N y $106^{\circ} 45^{\prime} 33.91^{\prime \prime}$ O). Este ecosistema natural se localiza en el municipio de Cuauhtémoc, Chihuahua, México. En la segunda fase del estudio se desarrolló el análisis parasitoscópico de los peces, en el laboratorio de Parasitología de la Facultad de Ciencias Químicas (FCQ) de la Universidad Autónoma de Chihuahua (UACH) campus II. Se capturaron 30 especímenes $(\mathrm{N}=30)$, los cuales se analizaron sin discriminación de especie, sexo, tamaño ni longitud. La captura se realizó en el periodo comprendido desde septiembre hasta noviembre de 2011 . El número de peces en el presente estudio garantiza la confiabilidad de los datos, ya que la recomendación para la valoración de helmintos es analizar por lo menos 15 ejemplares con el propósito de que la prevalencia sea confiable (Vidal Martínez et al., 2001). Una vez capturados, los ejemplares fueron transportados vivos en un contenedor especial que fue previamente adaptado con una bomba oxigenadora Elite 802 con el propósito de mantener los órganos intactos y evitar pérdida de características morfológicas de los parásitos.

En laboratorio, los animales fueron sacrificados y se procedió a realizar un examen post mortem, que consistió en una incisión longitudinal en la zona ventral, se removieron las vísceras del tórax, las abdominales y el sistema esquelético con el propósito de descubrir signos o lesiones de enfermedad. Se utilizó un microscopio binocular de campo claro (Carl 
Zeiss Primo Star) y un estereoscopio binocular con luz tipo LED (Zeigen). Para la disección se utilizaron tubos de ensayo, cajas Petri de plástico, portaobjetos, navajas de bisturí número 20 y soluciones fijadoras o conservadoras de alcohol-formol-ácido acético (AFA).

La disección inició con el corte del opérculo y extracción de branquias, que se colocaron en una caja Petri con solución salina fisiológica al $0.85 \%$ para ser observados en microscopio. Se realizaron tres cortes en la zona ventral del pez. El primero recorrió el vientre desde el ano hasta el espacio de las branquias. El segundo, lateral, desde la zona superior de una de las branquias hasta el ano y, finalmente, el tercero, desde el inicio de las dos aperturas anteriores, incluyendo la zona branquial. Se separó la piel quedando descubiertas las vísceras y se tuvo precaución de no realizar las incisiones demasiado profundas para no dañar órganos superficiales. Se separaron los órganos, cortando los tejidos que los unían y se colocaron en cajas Petri con una solución salina fisiológica al $0.85 \%$. La piel fue separada para examinar todos los órganos $y$, de esta manera, detectar posibles anomalías, parásitos internos, tumores, hemorragias y cualquier tejido anómalo.

\section{Análisis parasitoscópico}

De manera posterior a la disección y separación de órganos se extrajo una porción de epitelio intestinal mediante una incisión longitudinal del tracto gastrointestinal. La muestra resultante fue humedecida con una solución salina fisiológica al $0.85 \%$ con el fin de evitar su deshidratación. La obtención de la muestra se realizó con una hoja de bisturí No. 20 colocada en posición perpendicular y se raspó el epitelio intestinal de 6 a $8 \mathrm{~cm}$ de largo. La muestra fue analizada mediante microscopía. El análisis del tejido muscular se realizó mediante la técnica de triquinoscopía (OIE, 2008), también conocida como compresión en placas. Una vez obtenida la muestra, se colocó entre las placas de vidrio del compresor y se observó por medio de un estereoscopio con luz tipo LED (Zeigen) para la identificación de los parásitos tisulares. Al encontrar organismos parásitos, en una primera etapa se identificaron mediante morfometría $y$, en una segunda etapa, se procedió a su conservación en soluciones AFA.

\section{Frecuencia total parasitaria (FT) y frecuencia parasitaria por especie (FP)}

Se determinó la FT y la FP de acuerdo con la metodología propuesta por Vidal Martínez et al. (2001) y se utilizaron las siguientes ecuaciones:

$$
F T=\frac{\text { Número de especímenes infectados }}{\text { Número total de especímenes }} \times 100
$$

$F P=\frac{\text { Número de especímenes infectados por un género de Helminto }}{\text { Número total de especímenes }} \times 100$

\section{Soluciones conservadoras y técnica de tinción}

Se utilizaron dos emulsiones conservadoras; la primera fue una mezcla fijadora de AFA específica para platelmintos y la segunda una solución de AFA específica para nemátodos (De Haro et al., 2002). Se utilizaron tinciones para la identificación de nemátodos, tremátodos y céstodos. Las infusiones colorantes fueron preparadas con $\mathrm{KAl}\left(\mathrm{SO}_{4}\right)_{2}$ en $30 \mathrm{~g}$, así como colorante de alumbre de carmín $\left(\mathrm{C}_{22} \mathrm{H}_{20} \mathrm{O}_{13}\right)$ en polvo en $30 \mathrm{~g}$. Se mezclaron los $30 \mathrm{~g}$ de KAl$\left(\mathrm{SO}_{4}\right)_{2}$ con los $30 \mathrm{~g}$ de pigmento de $\mathrm{C}_{22} \mathrm{H}_{20} \mathrm{O}_{13}$ y se disolvieron en $200 \mathrm{ml}$ de agua destilada. Se hirvieron durante 1 $h$, se dejaron enfriar y se filtraron con un embudo de porcelana en un filtro Whatman número 1. El papel filtro con el sedimento se volvió a hervir con $200 \mathrm{ml}$ más de agua destilada durante 30 min y se volvió a filtrar. Los dos filtrados se mezclaron y se reintegró el volumen hasta $400 \mathrm{ml}$ con agua destilada.

Para la solución acuosa saturada de carbonato de litio $\left(\mathrm{Li}_{2} \mathrm{CO}_{3}\right)$ se agregó agua destilada hasta disolver la sal, se procuró que quedara un residuo del carbonato y se etiquetó. Para la emulsión aclaradora de Amman se colocaron en un mortero $20 \mathrm{~g}$ de fenol $\left(\mathrm{C}_{6} \mathrm{H}_{5} \mathrm{OH}\right)$ previamente fundidos en baño maría. Se agregaron $20 \mathrm{ml}$ de ácido láctico $\left(\mathrm{C}_{3} \mathrm{H}_{6} \mathrm{O}_{3}\right)$ y se mezclaron hasta obtener una mezcla homogénea sin grumos. Posteriormente se agregaron $40 \mathrm{ml}$ de glicerina $\left(\mathrm{C}_{3} \mathrm{H}_{8} \mathrm{O}_{3}\right)$ y se incorporaron $20 \mathrm{ml}$ de agua destilada (De Haro et al., 2002).

\section{Preparación de helmintos}

Los helmintos se lavaron en agua durante $24 \mathrm{~h}$ y por capilaridad se introdujo el fijador. Se colocaron en una solución de Amman por $24 \mathrm{~h}$ y se lavaron en agua. Los helmintos se colocaron en $\mathrm{C}_{2} \mathrm{H}_{6} \mathrm{O}$ al $50 \% 1 \mathrm{~h}$, se pusieron en agua destilada $10 \mathrm{~min}$ para su disposición en $\mathrm{C}_{2} \mathrm{H}_{6} \mathrm{O}$ al $70 \%$ durante $2 \mathrm{~min}$. Se colocaron en la solución de tinción de alumbre carmín por un periodo de 24 h y después en alcohol ácido acético al $3 \%$, hasta que se observó cierta definición en la tinción. Se dispusieron en $\mathrm{C}_{2} \mathrm{H}_{6} \mathrm{O}$ al $70 \%$ en un lapso de 2 min y posteriormente en una solución de $\mathrm{C}_{2} \mathrm{H}_{6} \mathrm{O}$ al $70 \%$ saturada con $\mathrm{Li}_{2} \mathrm{CO}_{3}$ 
durante $1 \mathrm{~h}$. Después se situaron en $\mathrm{C}_{2} \mathrm{H}_{6} \mathrm{O}$ al $95 \%$ por $1 \mathrm{~h}$. Los helmintos se colocaron en $\mathrm{C}_{2} \mathrm{H}_{6} \mathrm{O}$ con aceite de cedro $\left(\mathrm{C}_{15} \mathrm{H}_{26} \mathrm{O}\right)$ en proporción 50-50 durante $1 \mathrm{~h}$ $y$, finalmente, se montaron en resina sintética Entellan (De Haro et al., 2002). La identificación de helmintos se realizó con el apoyo de los atlas de Vidal Martínez et al. (2001), con Bunkley Williams y Williams (1995) y Hoffman (1999).

\section{RESULTADOS}

En los especímenes analizados se observó una FT de $90 \%$, lo que indica que en el ecosistema bajo estudio se presenta una fuerte parasitosis. Las especies encontradas (FP) fueron P. tomentosa con 83.33\%, Gyrodactylus spp. con $76.67 \%$ y B. acheilognathi con $6.67 \%$. En la Figura 1 se observa el tremátodo Gyrodactylus spp., identificado en una de las agallas de los peces en este estudio. En algunas fotografías fue posible observar su órgano de adhesión llamado haptor (Figura 2). Este tremátodo es monogéneo, de cuerpo alargado y presenta seis pústulas cefálicas a lo largo de la faringe, así como lóbulos cefálicos bien desarrollados, que a su vez tienen dos anclas medianas para su proceso de fijación (Hoffman, 1999). Este tremátodo fue observado en las agallas de la carpa común. Se fija en piel, aletas y puede ocasionar una infestación severa en branquias, por lo que es considerado como de gran importancia para la salud acuícola, ya que al aumentar su presencia impide el intercambio de gases en las branquias y disminuye la productividad de los peces (Bunkley Williams y Williams, 1995).

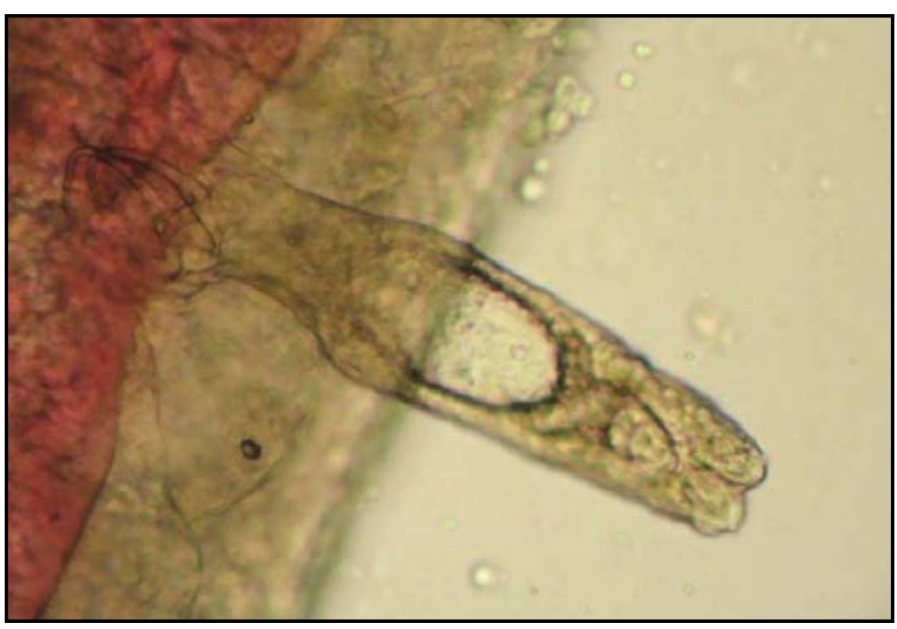

Figura 1. Tremátodo monogéneo del género Gyrodactylus sp. identificado en C. carpio (50X). Fotografía tomada por Martín Renato Hernández Castaños.

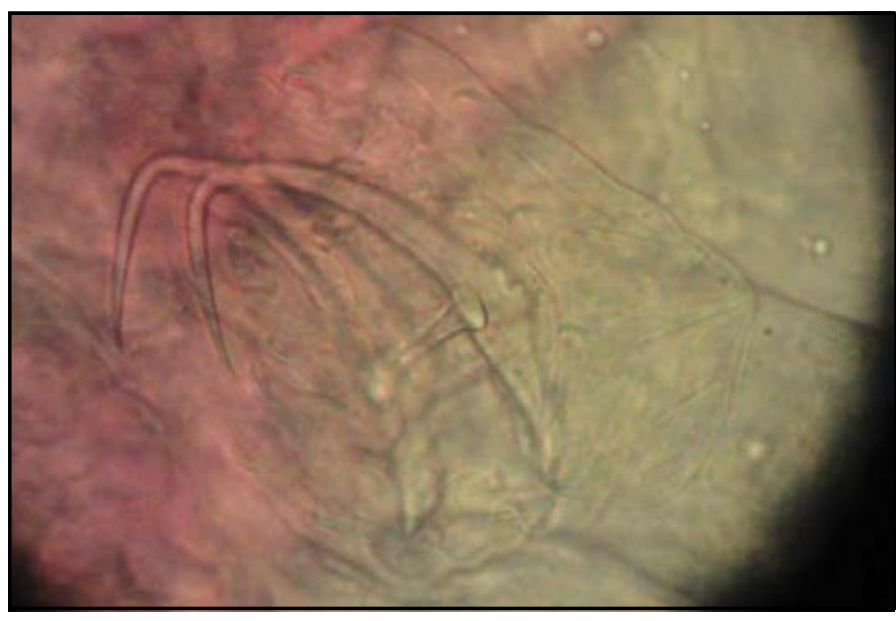

Figura 2. Órgano de adhesión (haptor) del tremátodo monogéneo Gyrodactylus sp. (65X). Fotografía tomada por Martín Renato Hernández Castaños.

El céstodo pseudofilídeo de la especie B. acheilognathi fue encontrado en este trabajo parasitando el epitelio intestinal. Se localizó en estado maduro, tal como se muestra en la Figura 3, mientras que en la Figura 4 se muestran los huevos no embrionados del mismo; céstodo asiático, introducido, cuyo hospedero definitivo es la carpa común (C. carpio) y cuyo sitio de infección es el tracto intestinal. Este céstodo está ampliamente distribuido entre las especies nativas del altiplano mexicano. Presenta un escólex en forma de corazón y los órganos de adhesión se localizan en este. $P$. tomentosa se encontró en el epitelio intestinal con una FP de $83.33 \%$. En esta investigación se localizaron hembras adultas, lo cual se visualiza en la Figura $5 \mathrm{y}$, en algunas ocasiones, fue posible observar la presencia de huevos en útero, como se muestra en

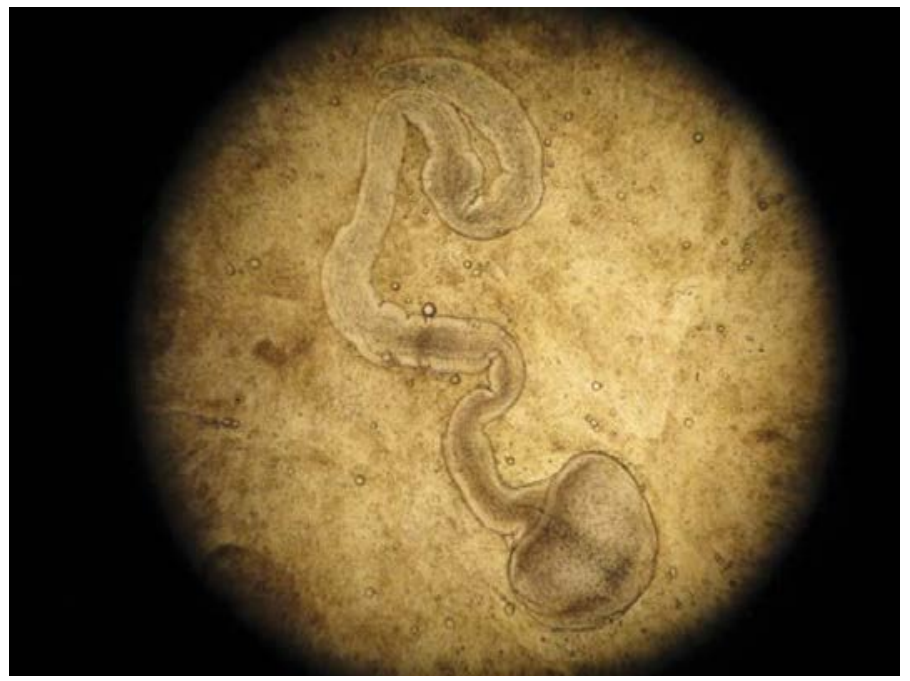

Figura 3. Detalle del céstodo adulto pseudofilídeo de B. acheilognathi (40X). Fotografía tomada por Martín Renato Hernández Castaños. 


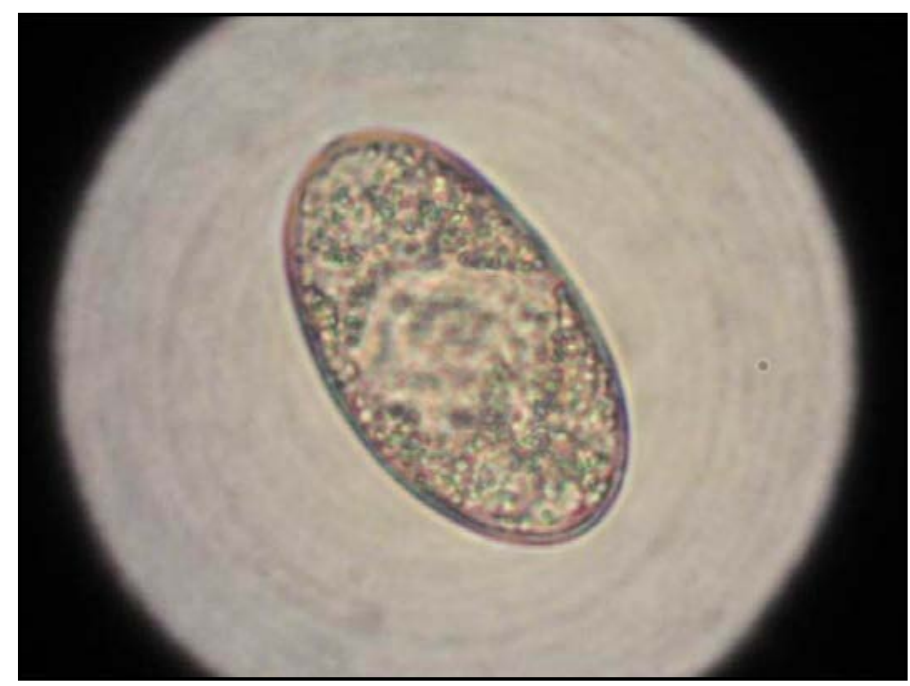

Figura 4. Huevo no embrionado de B. acheilognathi (65X). Fotografía tomada por Martín Renato Hernández Castaños.

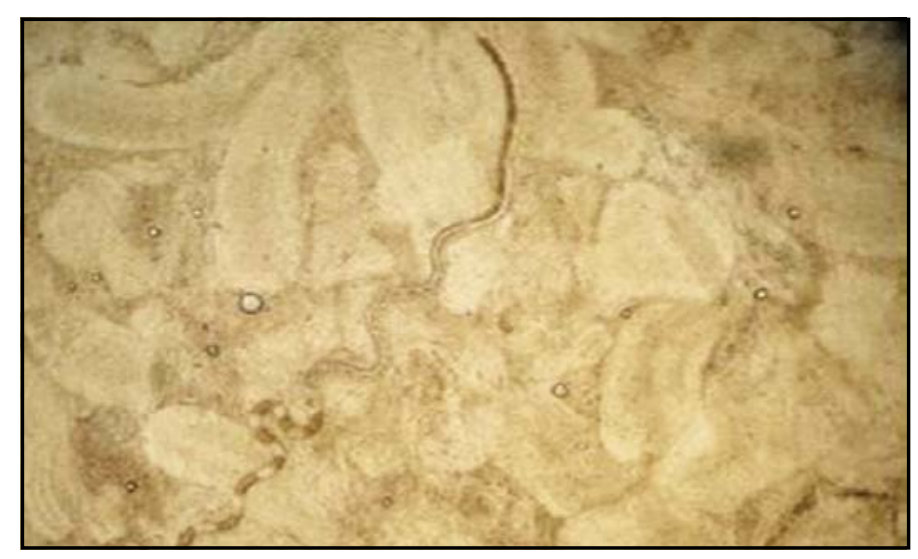

Figura 5. Hembra adulta de $P$. tomentosa en el epitelio intestinal (40X). Fotografía tomada por Martín Renato Hernández Castaños.

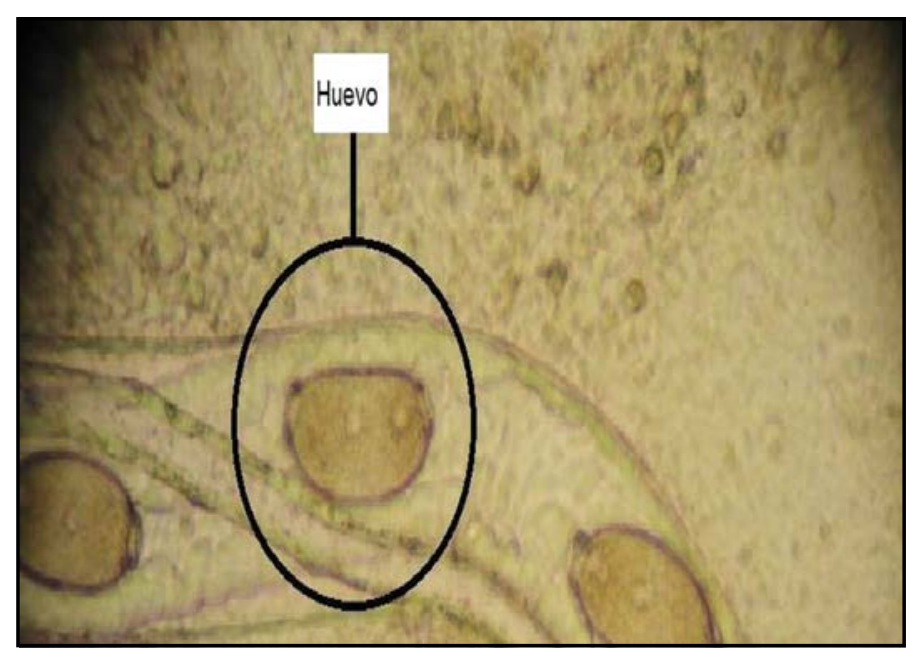

Figura 6. Huevos en útero de P. tomentosa (50X). Fotografía tomada por Martín Renato Hernández Castaños.

\section{IIVESEIGACIÓn

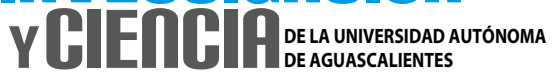

la Figura 6. Además, se encontraron machos, en los que fue posible observar la espícula copulatoria envainada.

\section{DISCUSIÓN}

En el ecosistema bajo estudio se encontró una FT de $90 \%$, en donde se identificó a P. tomentosa (83.33\%), a Gyrodactylus spp. (76.67\%) y, a B. acheilognathi (6.67\%). Es conveniente resaltar que ninguna de estas especies es considerada como zoonótica; es decir, no representan un riesgo para el humano, al menos, en el corto plazo. Se debe recordar que los parásitos representan un componente esencial en todo ecosistema acuático. En otras palabras, un ecosistema prístino puede albergar poblaciones sanas de parásitos que proceden de la biota de una determinada región (Salgado Maldonado, 2014b). Resulta claro que la presencia de parásitos puede ser un indicador de ciertos padecimientos en la ictiofauna, toda vez que algunos de ellos han sido responsables de enfermedades en peces. Por ejemplo, en ejemplares adultos de salmón pueden reducir la productividad hasta 55\% (Krkošek et al., 2012). Gyrodactylus se fija en la piel, aletas y branquias y puede ocasionar una infestación severa, impedir la respiración del hospedero o, en el peor de los casos, producir una infestación masiva y provocar su muerte debido a las lesiones en branquias (Harris et al., 2004). Su distribución se da ampliamente en México (Vidal Martínez et al., 2001).

En otro estudio, los investigadores Walberg et al. (2003) encontraron este parásito ampliamente distribuido en dos áreas de California y se ha reportado su aparición en ríos de Noruega, en donde puede representar un factor de riesgo para la producción de salmón. Otros estudios como el de García Vázquez et al. (2007) documentaron el parásito G. cichlidarum, el cual se encontraba afectando la producción de tilapia en el río Nilo. Este céstodo se encuentra prácticamente en todo el mundo (Marcogliese et al., 2001), también se localiza ampliamente distribuido entre las especies nativas de peces del altiplano mexicano. Se considera que posiblemente fue llevado a Europa y a los Estados Unidos de Norteamérica en el transporte de peces de ornato que arribaron de diferentes partes del mundo. También se cree que ha sido introducido a los diversos cuerpos de agua de México y se ha dispersado ampliamente en todo el país mediante prácticas de acuicultura poco cuidadosa. 
B. acheilognathi cuenta con un solo hospedero intermediario como lo es un copépodo con una larva procercoide. Las vellosidades intestinales son seriamente dañadas, puede provocar hemorragias, hemólisis o vesiculación de la lámina propia de la mucosa. Es causante de la muerte de peces jóvenes por anemia, obstrucción y estallamiento del intestino. Los peces adultos que son infectados no mueren y continúan alimentándose activamente; sin embargo, los cambios histopatológicos pueden llegar a ser serios, ya que afectan su crecimiento. Este parásito no es zoonótico y presenta un escólex en forma de corazón. Tiene unos órganos de adhesión característicos que se localizan en el escólex. Su distribución geográfica se da en los estados de Campeche, Michoacán, Tabasco y Yucatán y su origen es Asia Oriental (Vidal Martínez et al., 2001).

B. acheilognathi fue identificado por primera vez en Japón y después se extendió en todos los continentes (Hansen et al., 2006), llegó a los Estados Unidos de Norteamérica, en donde ha afectado a las especias nativas. También es considerado como uno de los helmintos más peligrosos para peces de cultivo y, a su vez, de riesgo potencial para las poblaciones silvestres de peces en todo el mundo. La patología que causa es grave, principalmente en larvas y ejemplares juveniles de peces (Vázquez Núñez et al., 2004). Velázquez Velázquez (2015) encontró altos niveles de este céstodo parasitando la especie Profundulus candalarius en un ecosistema del estado de Chiapas, México.

Con respecto al género Pseudocapillaria que se encontró parasitando el epitelio intestinal, se debe especificar que es endémico de Eurasia y de Norteamérica, pero ha sido reportado en Gran Bretaña, Finlandia, Dinamarca, Francia y Alemania. Está bien documentado que los parásitos de esta clase de metazoarios se mantienen en sistemas cerrados. Este nemátodo tiene una amplia gama de hospederos (Pazooki et al., 2011), por ejemplo, afecta a peces de la familia Cyprinidae, así como a otros miembros de diferentes órdenes. Es importante mencionar que el nivel de parasitismo en un ecosistema es reflejo del grado de contaminación que presenta el mismo (Galli et al., 2001; Salgado Maldonado, 2006).

En el caso específico de la Laguna de Bustillos y de acuerdo con los resultados de este estudio, se puede establecer que se ha reducido el número de especies de fauna que habitaban 0 que tradicionalmente se presentaban en este cuerpo de agua y, como consecuencia, se ha reducido también la variabilidad de los parásitos que aportaban dichas especies. Resulta importante mencionar que cualquier parasitosis es reguladora natural de poblaciones, tamaños y especies de peces; por lo cual no debe pensarse en exterminarlas, ya que son parte de un ciclo de vida, el cual puede afectar toda la cadena de la que forman parte.

CONCLUSIONES

La mayoría de los especímenes capturados presentaron los parásitos Gyrodactylus spp., B. acheilognathi yP. tomentosa. Es importante mencionar que aunque no se encontró una diversidad parasitaria distintiva, los parásitos fueron imperecederos y se puede inferir cierto grado de contaminación del ecosistema. Se sugiere a los potenciales consumidores que realicen la evisceración al momento de la captura, con la cual se evita la migración de parásitos a músculos. Además, es recomendable no arrojar los órganos al agua, debido a que de esta manera es posible que se infecten otros peces y animales que consuman dichos órganos.

LITERATURA CITADA

- BeCerril, M. A. Parasitología Médica. 2 ed. México: Editorial McGraw-Hill Interamericana, 10-11, 2008.

- BUNKLEY WILLIAMS, L. y WILLIAMS, E. H. Parásitos en peces de valor recreativo en agua dulce en Puerto Rico. Mayagüez, Puerto Rico: Departamento de Recursos Naturales y Ambientales de Puerto Rico y Departamento de Ciencias Marinas, 1995.

- DE HARO, A. I. et al. Diagnóstico morfológico de las parasitosis. 2 ed. D. F., México: Méndez Editores, 2002.
- GALLI, P. et al. Water quality as a determinant of the composition of fish parasite communities. Hydrobiologia, 452(1): 173-179, 2001

- GARCÍA VÁZQUEZ, E. et al. Alternative Mating Strategies in Atlantic Salmon and Brown trout. Journal of Heredity, 92 (2): 146-149, 2007.

- HARRIS, P. D. et al. Nominal species of the genus Gyrodactylus von Nordmann 1832 (Monogenea: Gyrodactylidae), with a list of principal host species. Systematic Parasitology, 59(1): 1-27, 2004. 
- HOFFMAN, L. G. Parasites of North American Freshwater Fishes. 2 ed. US: Cornell University, 1999.

- KINKELIN, P. et al. Tratado de las enfermedades de los peces. Zaragoza, España: Editorial Acribia, 1991.

- MARCOGLIESE, D. J. et al. Potential impacts of clearcutting on parasites of minnows in small boreal lakes. Folia Parasitologica, 48(4): 269-274, 2001.

- PAZOOKI, J. et al. Parasitic infection of an endemic fish (Blicca bjoerkna) and an exotic fish (Hemiculter beucisculus) in Anzali Lagoon, Caspian Sea, Iran. Iran Journal of Parasitology, 6(3): 66-73, 2011.

- RUBIO, A. H. et al. Are crop and range land being contaminated with cadmium and lead in sediments transported by wind from an adjacent contaminated shallow lake? In J. F. Martin Duque, C. A. Brebbia, D. E. Emmanouloudis \& U. Mander (Eds.), GeoEnvironment and Landscape Evolution II (pp. 135-141). WitPress, 2006.

- RUBIO, A. H. et al. Water quality in the Laguna de Bustillos of Chihuahua, Mexico. In M. de Conçelcao Cunha \& C. A. Brebbia (Eds.), Water Resources Management III (pp.155-160). Algarve, Portugal: WitPress, 2005.

- SALGADO MALDONADO, G. Checklist of helminth of freshwater fishes from Mexico (Zootaxa 1324). Auckland, New Zealand: Magnolia Press, 357 pp., 2006.

- SAlgado maldonado, G. et al. Helmintos parásitos de los peces del río Lacantún en la reserva de la biósfera Montes Azules, Chiapas. Nuevo León, México: Universidad Autónoma de Nuevo León, 142 pp., 2014.

- SALGADO MALDONADO, G. et al. Range extension of helminth parasites of Profundulus spp. (Teleostei: Profundulidae) from southern Mexico and Central America. Check List The Journal of Biodiversity Data, 10(6): 1507-1513, 2014.

- SLIFKO, T. R. et al. Emerging parasite zoonoses associated with water and food. International Journal of Parasitology, 30(1213): 1379-1393, 2000.
- soto, CH. G. Evaluación de parámetros fisicoquímicos y contenido de metales en sedimentos de la Laguna de Bustillos en Chihuahua, México. Tesis de Maestría. Chihuahua, México: Universidad Autónoma de Chihuahua, 2011.

- VIDAL MARTíneZ, V. M. et al. Atlas of the Helminth Parasites of Cichlid Fish of Mexico. Praga: Academia, 91-100, 2001.

- WALBERG, M. P. et al. Occurrence of Gyrodactylus perforates (Monogenea) on its fish host clevelandia (Gobildae) from bodega Bay and Tomales Bay, California. Bulletin of the Southern California Academy of Sciences No. 102. 2003.

\section{De páginas electrónicas}

- FAO (FOOD AND AGRICULTURE ORGANIZATION OF THE UNITED NATIONS). Fishery and Aquaculture 2007. 2007. Recuperado el 22 de noviembre de 2012, de http://faostat.fao.org

- HANSEN, S. P. et al. Experimental infection of the endangered bonytail chub (Gila elegans) with the Asian fish tapeworm (Bothriocephalus acheilognathi): impacts on survival, growth and condition. Canadian Journal of Zoology, 84(10): 13831394, 2006. doi: 10.1139/z06-126

- KRKOŠEK, M. et al. Impact of parasites on salmon recruitment in the Northeast Atlantic Ocean. Proceedings of the Royal Society B, 2012. Recuperado el 24 de junio de 2013, de http:// royalsociety.org/news/2012/parasites-impact-salmon/

- Ole (ORganización INTERnacional de ePIZOOtIAS). Triquinelosis. Manual de la OIE sobre animales terrestres. 2008. Recuperado de http://web.oie.int/esp/normes/mmanual/ pdf_es_2008/2.01.16.\%20Triquinelosis.pdf

- VÁZQUEZ NÚÑEZ, R. et al. Lesiones causadas por helmintos del aparato digestivo en peces estuarinos de la laguna Tres Palos, Guerrero, México. Veterinaria México, 35(4): 369-378, 2004. Recuperado el 3 de mayo de 2012, de http://www.ejournal. unam.mx/rvm/vol35-04/RVM35409.pdf

- VeLÁZQUeZ VELÁZQUeZ, E. The invasive tapeworm Bothriocephalus acheilognathi Yamaguti, 1934 in the endangered killifish Profundulus candalarius Hubbs, 1924 in Chiapas, Mexico. Bioinvasions Records, 4(4): 265-268, 2015. doi: http://dx.doi.org/10.3391/bir.2015.4.4.06 